\title{
MONITORING INLAND WATERS WITH THE APEX SENSOR, A WAVELET APPROACH
}

\author{
Knaeps $^{l}$, E., Raymaekers ${ }^{1}$, D., Sterckx ${ }^{1}$, S., Bertels ${ }^{1}$, L., Odermatt $^{2}, D$. \\ VITO - Flemish Institute for Technological Research \\ RSL - Remote Sensing Laboratories, University of Zurich
}

\begin{abstract}
In this study a new curve fitting approach is presented to derive TSM, CHL and CDOM concentrations in inland and coastal waters from water leaving-reflectance spectra. The approach is based on the wavelet transform and is tested on simulated water-leaving reflectance spectra. For simulations SIOPS and water concentrations, representative for the Scheldt river, were used. The results shown that the approach is less sensitive to errors in the atmospheric correction or specific sensor noise. The idea is based on the development of a new minimization criteria for curve fitting. Instead of minimizing the difference between modeled and measured spectra using a simple RMSE, the RMSE is now combined with specific wavelet features. Several types of errors and noise are added to the simulated spectra to find robust features. Two minimization criteria were found which are almost insensitive to a white error and less sensitive to adjacency effects.
\end{abstract}

Index Terms - wavelets, water quality, curve fitting, APEX, Scheldt

\section{INTRODUCTION}

Inland and coastal waters are optically complex because of the presence of Algae, Total Suspended Matter (TSM) and coloured dissolved organic matter (CDOM) and their combined influence on the water-leaving reflectance spectra. This implicates that several standard algorithms in use for water quality retrieval from the open ocean are not suited for these waters. With the advent of new sensors with improved spectral, spatial and radiometric resolution, new improved algorithms and approaches have been developed to retrieve water quality parameters in these complex waters. They range from simple site-specific empirical to purely analytical algorithms. In the analytical approach the water constituent concentrations are physically related to the measured reflectance spectra using sophisticated radiative transfer models (e.g. Hydrolight). These radiative transfer models are being used to generate Look-Up-Tables or train sophisticated neural networks [6] to retrieve concentrations values. The semi-analytical approach uses simplified bio- optical models. These models are then inverted (e.g. matrix inversion [4] or curve fitting [5]) to derive concentration values.

These analytical and semi-analytical approaches are based on our physical knowledge of the radiative transfer in waters and should be more robust and more widely applicable than the empirical counterparts. In practice these algorithms seem to be highly sensitive to errors in the atmospheric correction and sensor noise. Under the same conditions empirical band ratio or band difference algorithms sometimes have surprisingly good results. For instance, a curve fitting may be unsuccessful due to a mismatch in magnitude although the spectral shape may be correctly reproduced. In this case an empirical band difference algorithm will be insensitive to the white error.

To overcome these issues a new curve fitting technique is introduced. The new technique is based on the wavelet transform method. It allows to concentrate on different scales of the spectra. By weighing the different scales, different aspects of the spectral differences (shape, magnitude) can be taken into account. For this study simulated APEX (Airborne Prism Experiment) spectra are being used to develop the algorithm. The final goal is to implement the algorithm into the APEX processing chain for the generation of level 3 water quality products.

\section{STUDY AREA}

Our study area is part of the brackish lower sea Scheldt, situated between the city of Antwerp and the border of Belgium and the Netherlands. This zone corresponds to the zone of high turbidity [2].

\section{METHODOLOGY}

\subsection{Field Campaign}

On 23/06/2009 an intensive field campaign was organized on the river Scheldt simultaneously with an APEX airborne image acquisition. Water was sampled from vessels and pontoons ca. $50 \mathrm{~cm}$ below the water surface. These were 
used for concentration measurements and to analyze the inherent optical properties in the lab. At discrete points a BB-3 backscatter meter (Wetlabs.Inc.) was used.

\subsection{Specific inherent optical properties}

Water samples, taken during the field campaign were analyzed in the lab for their component concentrations and optical properties. The specific absorption spectra of particles, non-algae particles and phytoplankton were measured using a LICOR integrating sphere attached to an ASD spectrometer following the methods described by Tassan and Ferrari [8] and REVAMP protocols [9]. To retrieve the CDOM absorption coefficient of the water samples, the beam attenuation of the filtered water was measured with Ocean Optics equipment in a transparent cuvet. Specific backscattering for the TSM was retrieved from the $B B-3$ measurements.

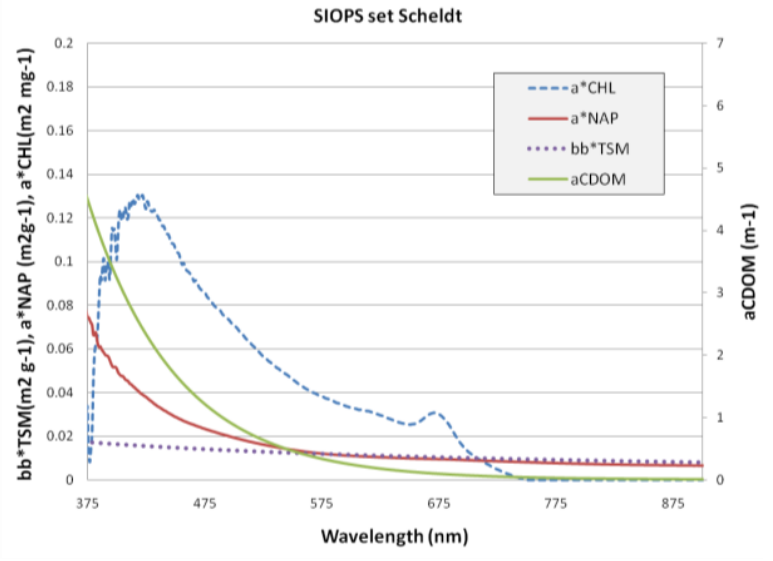

Figure 1: SIOP set for the Scheldt

\subsection{Simulations}

For this paper the water-leaving reflectance spectra were obtained through simulations with HYDROLIGHT and the bio-optical model of Albert and Mobley [1]. As an input the measured variation in TSM, CHL and CDOM concentrations and the SIOPS parameter set shown in Figure 1 were selected. The simulated spectra were then resampled to the APEX wavelengths.

\subsection{Wavelet analysis}

The wavelet transform is a mathematical function that provides a wavelength-frequency representation of the water-leaving reflectance spectra. It subdivides the original spectra into different scale components each corresponding to a certain frequency range. Each scale can be represented with respect to the original wavelengths and features in each scale correspond to particular wavelength regions. Having the data in a new representation reveals more information that is somehow hidden in the original spectra. For this study the HAAR wavelet transform was applied on the simulated spectra. The different scales of the wavelet were inspected.

The wavelet approach was tested in a curve fitting procedure (Figure 2). In this procedure TSM, CHL and CDOM concentrations $(\hat{C})$ are estimated by minimizing the error between modeled $(\hat{R})$ and measured $(R)$ spectra. In this approach the model of Albert and Mobley [1] was used in the forward calculations. The measured spectra $(R)$ are replaced by the simulations (Albert and Mobley [1] or Hydrolight) to have full control over the inputs. The algorithm starts with a set of initial concentrations values. The optimizer then calculates the RMSE between simulated $(R)$ and modeled $(\hat{R})$ spectra and subsequently adjusts the input concentrations $(\hat{C})$ until a minimum RMSE is obtained. The wavelets can be incorporated in this procedure by combining the original RMSE with specific wavelet scales and features.

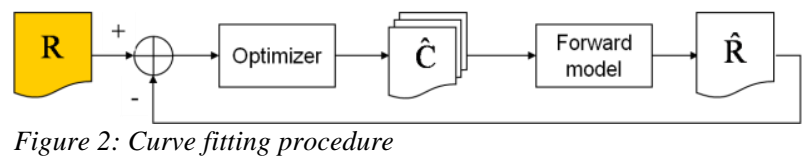

To select robust wavelet scales and features different types of errors were added to the simulated spectra and these were wavelet transformed. Robust features should be insensitive to these errors. In a first stage a white error, random noise, APEX specific sensor noise, adjacency effects and spectral calibration errors were added to the simulated spectra (Figure 3). Adjacency effects were simulated by making linear mixtures of the original water spectra (Remote sensing reflectance) and a vegetation spectrum. In a next step extra atmospheric errors will be introduced.

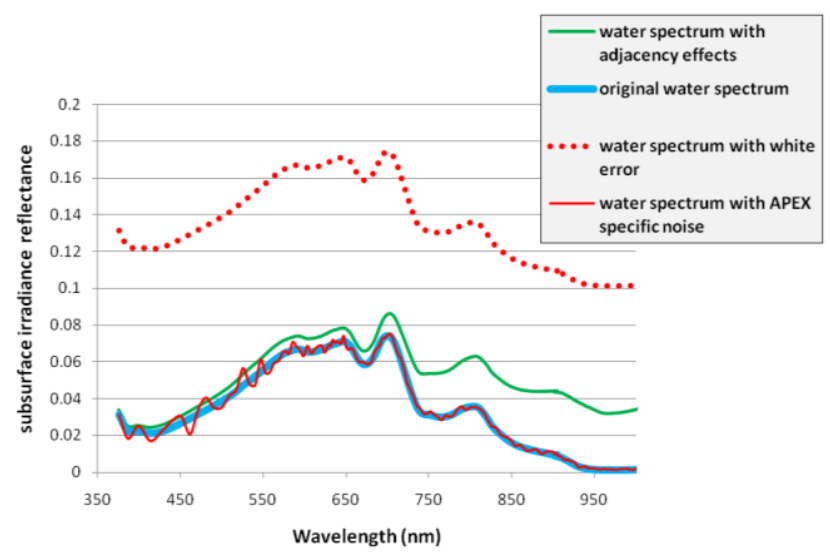




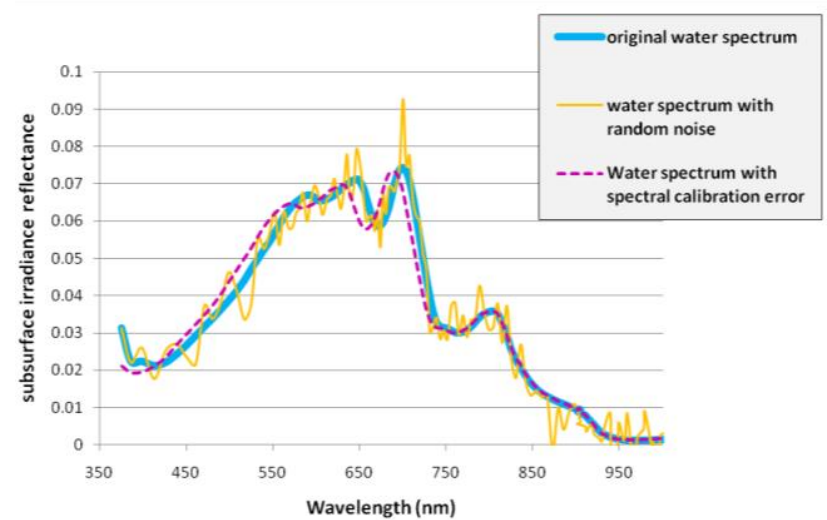

Figure 3: Simulated water spectra including errors

\section{RESULTS AND DISCUSSION}

The standard minimization procedure (using the RMSE as minimization criteria) is first tested for the simulations based on the Albert and Mobley [1] and the simulations based on Hydrolight before introducing errors. The model of Albert and Mobley [1] is also used in the forward simulations such that the input and estimated SPM concentrations match perfectly. Based on the Hydrolight simulations, the estimations perform almost equally well. Similar results were found for the CHL and CDOM concentrations. These results are further used as a reference for the wavelet analysis.

After careful inspection of the original spectra and the spectra including errors in the wavelet domain two new minimization criteria were found. The new criteria combine the orginal RMSE with two weighted features in the wavelet domain. Using the first new minimization criterion the algorithm is insensitive to a white error. The second criterion is less sensitive to adjacency effects compared to the orginal RMSE. Both the orginal RMSE as the new minimization criteria were insensitive to random noise, APEX specific sensor noise and spectral calibration errors.

The performance of minimization criteria 1 is shown in Figure 4 for the estimation of TSM, CHL and CDOM concentration. The performance of minimization criteria 2 is shown in Figure 5. In Figure 4 the model of Albert and Mobley [1] is used as reference such that the standard curve fitting (green squares) works perfectly. In Figure 5 hydrolight is used as reference. The red triangles show the performance of the standard algorithms (using a simple RMSE as minimization criteria) when adding errors to the simulated spectra. In Figure 4 a white error of 0.1 is added, in Figure 5 adjacency is added to the original spectra. The yellow squares show the performance of the new criteria (combining the original RMSE with wavelet features) without adding errors. The blue diamonds represent the results for the new criteria with errors.

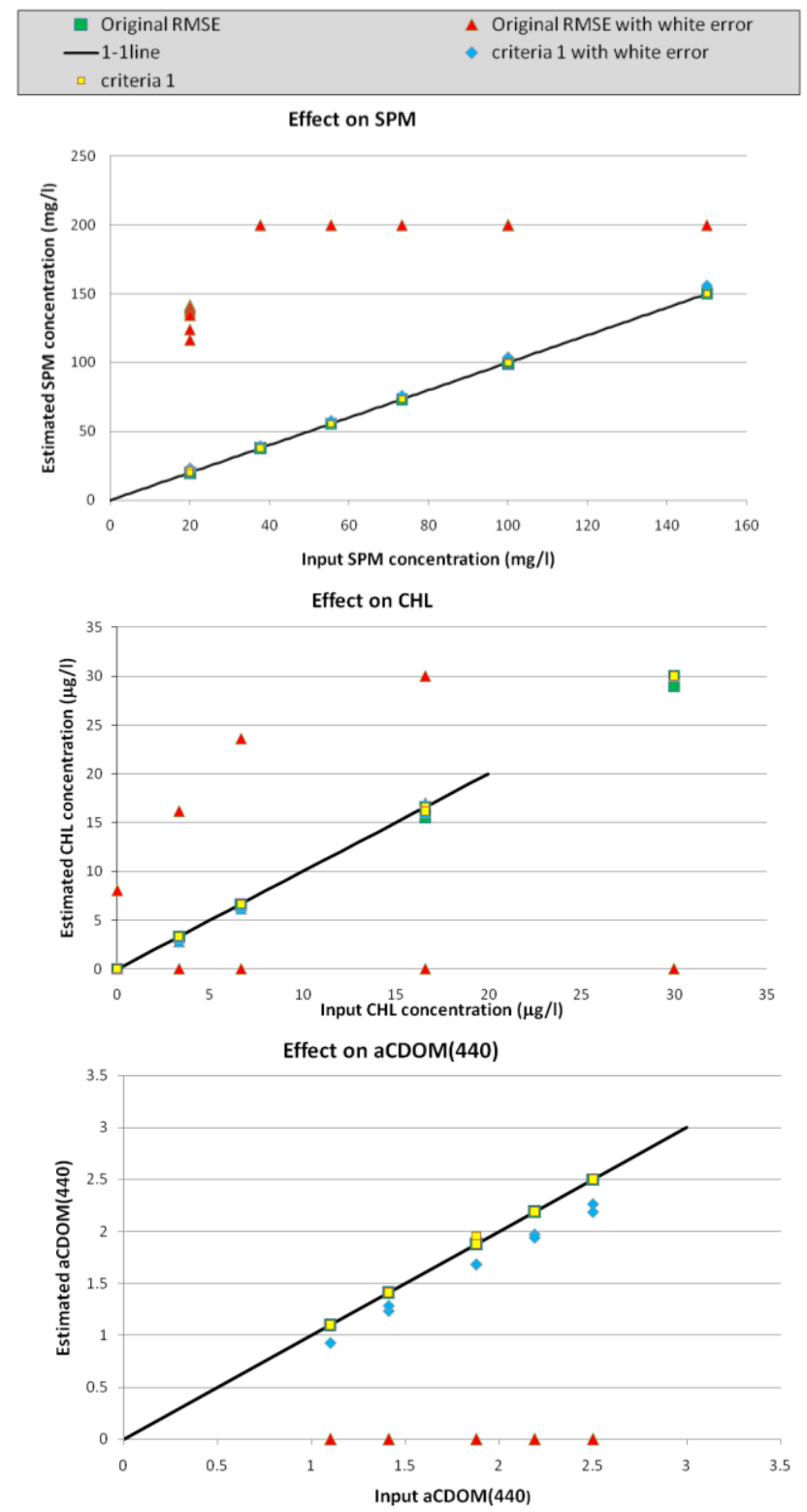

Figure 4: Effect of a white error on the estimation of SPM, CHL and CDOM concentration

Clearly the standard curve fitting is highly sensitive to white errors and adjacency effects. SPM concentrations reach the upper bounds of the algorithm (set to $200 \mathrm{mg} / \mathrm{l}$ ), CHL concentrations are either over or underestimated and $\operatorname{aCDOM}(440)$ is 0 . The new minimization criteria seem to be much more robust then the standard RMSE. The first criteria (Figure 4) is almost insensitive to the white error, only $\operatorname{aCDOM}(440)$ is slightly underestimated. Criteria 2 (Figure 5) is still influenced by the adjacency effects but the 
results are significantly improved compared to the orginal standard algorithm.

\begin{tabular}{l}
$\begin{array}{l}\text { Original RMSE } \\
\text { criteria } 2 \text { with adjacency effects } \\
\text { criteria 2 }\end{array}$ \\
\hline
\end{tabular}
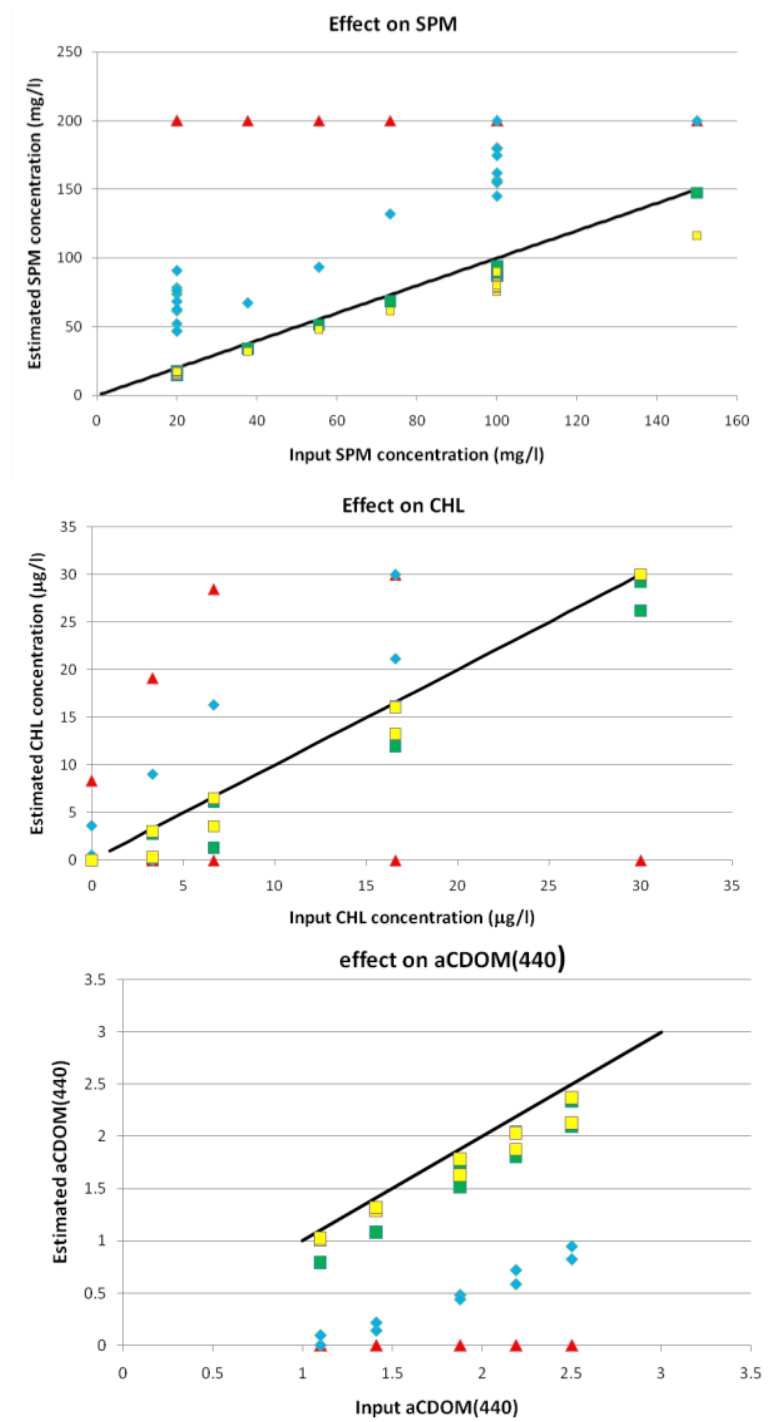

Figure 5: Effect of adjacency effects on the estimation of SPM, CHL and CDOM concentration

These first results show that the curve fitting approach can be made more robust (i.e. insensitive to specific error sources) by adding wavelet features in the minimization criteria. This suggests that the new algorithm might perform better then the traditional approach when applying it to real data. This hypothesis will be tested on calibrated APEX imagery acquired over the Scheldt river and two inland lakes (Spuikom and Lake Constance), available in the second quarter of 2010.
Up till now no single minimization criteria was found that is insensitive to both white errors and adjacency effects. This implies that a first inspection of the data might be needed to judge on the source of errors and that the minimization criteria should be selected accordingly. For adjacency errors the adjacency detection method based on the Near Infrared similarity spectrum [7] can be used.

Further research includes the addition of atmospheric errors (e.g. wrong estimate of visibility or wrong aerosol model) to the simulated spectra, testing the sensitivity of the newly developed minimization criteria to this errors, or if needed develop new minimization criteria. The performance of this new technique for the retrieval of water quality parameters from APEX spectra will be compared to the results of other hyperspectral inversion approaches.

\section{REFERENCES}

[1] Albert, A and C. Mobley, "an analytical model for subsurface irradiance and remote sensing reflectance in deep and shallow case-2 waters," opt. express 11, 2873-2890, 2003

[2] Baeyens, W., B. van Eck, C. Lambert, R. Wollast and L. Goeyens, "General description of the Scheldt estuary", Hydrobiologia, 366: 1-14, 1998

[3] Gordon, H. R., Brown, O. B., Jacobs, M.M., "computed relationship between the inherent and apparent optical properties of a flat homogeneous ocean". applied optics 14(2), pp. 417-427,1975

[4] Hakvoort, H., J. De Haan, R. Jordans, R. Vos, S. Peters, and M. Rijkeboer, "Towards airborne remote sensing of water quality in The Netherlands - validation and error analysis", ISPRS Journal of Photogrammetry \& Remote Sensing, 57:171-183, 2002

[5] Keller, P.A., "Comparison of two inversion techniques of a semianalytical model for the determination of lake water constituents using imaging spectrometry data", Sci Total Environ, 14;268(1-3):189-96, 2001

[6] Schiller, H. and Doerffer, R., "Improved determination of coastal water constituent concentrations from MERIS data", IEEE Transactions on Geosciences and Remote Sensing 43, pp. 1585-1591, 2005

[7] Sterckx, S., Knaeps, E., Ruddick, K, "Detection and Correction of Adjacency Effects in Hyperspectral Airborne Data of Coastal and Inland Waters: the Use of the Near Infrared Similarity Spectrum”, International Journal of remote sensing, accepted

[8] Tassan, S., Ferrari, G. M., "an alternative approach to absorption measurements of aquatic particles retained on filters", limnol. oceanogr. 40: $1358-1368,1995$

[9] Tilstone G.H, G.F.Moore, K. Sörensen, R. Doerffer, R. Rottgers, K.G. Ruddick, R. Pasterkamp and P.V. Jorgensen, "regional validation of meris chlorophyll products in north sea coastal waters", revamp inter-calibration report, 2002 